\title{
Osteogênese Imperfeita em cão jovem da raça pinscher
}

\author{
Osteogenesis Imperfect in an Young Pinscher Dog \\ Paula Priscila Correia Costa ${ }^{1,2}$, Juliana Custódio ${ }^{2}$,Fernanda Sayuri Ebina ${ }^{2}$, Liliane Lima da Silva ${ }^{2}$, \\ Pedro Ernesto da Cunha ${ }^{2}$, Ruben Horn de Vasconcelos ${ }^{2}$, Wesley Lyeverton Correia Ribeiro ${ }^{3} \&$ Luana Azevedo de Freitas ${ }^{2}$
}

\begin{abstract}
Background: Osteogenesis imperfecta is a severe genetic disease rarely described in veterinary medicine. This multisystemic condition is caused by a defect in the production and metabolization of collagen, which implicates in bone fragility. This disease has been described in cattle, sheep, domestic felines, mouse and dogs of different breeds, including collie, golden retriever, beagle, dachshund and chow chow. Animals affected by this condition present multiple fractures without previous occurrence of trauma. Therefore, this report aimed to describe a case of osteogenesis imperfecta type III in a miniature pinscher dog.

Case: A 1-year-old male miniature pinscher dog, was admitted for clinical evaluation in Fortaleza, Brazil, with a history of spontaneous fractures without known causes. This animal was maintained indoors, fed on dry feed and presented recurrent events of claudication and pain. In the physical examination, the individual walked solely with the forelimbs, avoiding the use of the hind limbs and presented pain behavior. Bulging of the skull was observed laterally, which promoted a triangular appearance of head and face. Fontanelles were soft, and the eyes presented blueish sclera and corneal opacity. Teeth were small, translucid, fragile and deformed. Radiography images revealed bulging of the calvaria and persistent fontanelles with open cranial sutures. In addition, cranial convolutions were less clear, which was compatible with hydrocephalus. Dental roots were narrow, short and presented partial pulp obliteration. The radiographic contrast of the dentin was low with a reduction of periapical radiolucency. Bone radiopacity was low in the bones of the abdomen and pelvis, in addition to femur. Metaphysis of the right tibia was enlarged and angular. Multiple fractures were identified in the pelvis with the formation of opaque bony calluses and bone marrow sclerosis. Physiological parameters and blood test results were unaltered. After the diagnosis of osteogenesis imperfecta type III, treatment was performed with meloxicam $0.1 \mathrm{mg} / \mathrm{kg}$, SID, VO) and tramadol ( $2 \mathrm{mg} / \mathrm{kg}$, TID, VO) until pain was controlled. When the patient was stable, the treatment was halted until the next event of fractures followed by pain.

Discussion: Type I collagen is considered the most abundant protein in connective tissue, accounting for $95 \%$ of the various types of collagen found in bone tissue. In osteogenesis imperfecta, there is a shortage in coding of type I collagen, which prevents this protein from exerting its structural functions properly. In addition, it is subjected to intra and extracellular degradation, affecting cell migration and differentiation and, concomitantly, cell-matrix interaction. Hence, these changes are considered the main pathophysiological factors of osteogenesis imperfecta. There is a wide range of phenotypic presentations in individuals with osteogenesis imperfecta. However, there is a pattern of fractures and features that aid clinically and radiologically in the characterization of the disease and form the basis for case identification and diagnosis. In this case, the diagnosis was concluded primarily considering significant clinical signs, such as: repetitive fractures, triangular face, bulging of the skull, bluish sclera and corneal opacity. Radiographic examinations were used to confirm the diagnosis and to aid in treating the fractures. Although the genetic test provides a better understanding of the disease, it was not performed in this case, as it is routinely unavailable in many treatment centers. In conclusion, we described a case of osteogenesis imperfecta in a miniature pinscher dog.
\end{abstract}

Keywords: bone fragility, defect of collagen type I, imperfect dentin-genesis.

Descritores: fragilidade óssea, defeito do colágeno tipo I, dentinogênese imperfeita. 


\section{INTRODUÇÃO}

A osteogênese imperfeita (OI) é uma doença genética rara, grave e pouco descrita na medicina veterinária causada por defeito na produção e metabolização do colágeno. É uma enfermidade multissistêmica que está frequentemente associada a fragilidade óssea, acompanhada de múltiplas fraturas com ausência de trauma ou traumatismo mínimo. A causa predominante de OI é resultante de mutações autossômicas dominantes nos genes que codificam o colágeno tipo I (COL1A1 e COL1A2) [16]. A doença foi descrita anteriormente em bovinos [2], ovinos [4], felinos domésticos [24], camundongos [10], tigres [12] e cães das raças Collie [17], Golden Retriever [8], Beagle [9] e Dachshund [18]. Segundo Campbell et al. [8], a OI canina e humana parecem homólogas em termos de apresentação clínica, etiologia e patogênese.

O presente relato de caso tem por objetivo descrever a ocorrência de osteogênese imperfeita em um cão da raça pinscher de um ano.

\section{CASO}

Um cão da raça Pinscher, macho, de 1 ano de idade, foi atendido em um consultório veterinário situado na cidade de Fortaleza, CE, com histórico, desde o nascimento, de fraturas espontâneas sem causa definida, com hábitos ativos e vivência em ambiente interno. Alimentava-se de ração seca e apresentava quadros recorrentes de claudicação e dor.

No momento do atendimento, o paciente apresentava-se alerta, locomovendo-se, apenas, com as patas dianteiras (não apoiando no chão as patas traseiras) e com episódios de dor. No exame físico, o paciente apresentava-se normohidratado, normocorado, com frequência cardíaca e temperatura normais. No entanto, apresentava crânio abaulado lateralmente conferindo a cabeça e face o aspecto triangular (Figura 1) e com fontanelas abertas, esclera azulada (Figura 2) e opacidade de córnea, dentes pequenos, translúcidos, acinzentados, frágeis e deformados (figura 3). Foram requisitados exames radiográficos de crânio e pelve além de ultrassonografia, hemograma completo e dosagens bioquímicas de uréia, creatinina, cálcio, ALT/ TGP, fosfatase alcalina e GGT.

O exame radiográfico do crânio (Figura 4) revelou, na incidência lateral, abaulamento da calvária e adelgaçamento da cortical com fontanelas persistentes e suturas cranianas abertas. Além de, aspecto homogêneo da calvaria, devido à perda das marcações normais das convolações cranianas, achados compatíveis com hidrocefalia. As raízes dentais eram estreitas e curtas, com obliteração pulpar parcial, concomitante à diminuição do contraste radiográfico da dentina, com redução da radiolucidez periapical. A incidência ventrodorsal (Figura 5) de abdome, pelve e fêmur identificou decréscimo generalizado na radiopacidade óssea, com adelgaçamento da cortical. Observou-se, na tíbia direita, a metáfise alargada e angulada. Por conseguinte, na pelve, fraturas múltiplas observadas em nível de asa do íleo direito, corpo do ísquio esquerdo e corpo do ísquio direito, com formação de calos ósseos opacos e esclerose medular.

No exame ultrassonográfico do crânio houve a visibilização de ventriculomegalia (ventriculos lateral direito e esquerdo) cerebral devido a dilatação por conteudo liquido anecoico. Presença de discreta quantidade de liquido anecoico em terceiro ventriculo (discreta dilatação) e espessamento de superficies de ventriculos, corroborando com os achados radiográficos de hidrocefalia. Os demais órgãos visibilizados apresentam-se dentro dos parâmetros normais.

No hemograma completo não foram observadas alterações e nos exames de bioquímica, um discreto aumento sérico na dosagem de cálcio $(12,32 \mathrm{mg} / \mathrm{dL})$.

Após o diagnóstico clínico e radiográfico de osteogênese imperfeita classe III, foi realizado tratamento paliativo, composto por meloxicam (Maxicam $\left.0,5 \mathrm{mg}^{\circledR}\right)^{1}\left[0,1 \mathrm{mg} \cdot \mathrm{kg}^{-1}\right.$ PO q24 h durante 7 dias $] \mathrm{e}$ Cloridrato de tramadol (Nulli ${ }^{\circledR}{ }^{1}\left[2 \mathrm{mg}^{-k^{-1}} \mathrm{PO}\right.$ q8 h durante 7 dias] até cessar os episódios de dor, devido às múltiplas fraturas. $\mathrm{O}$ tratamento era descontinuado quando o animal estava estabilizado. Contudo, retornado, quando do surgimento de novos episódios de fraturas, seguidos de dor.

\section{DISCUSSÃO}

O colágeno do tipo I é considerado a proteína mais abundante dos tecidos conjuntivos [3], e representa $95 \%$ dos diversos tipos de colágeno encontrados em tecido ósseo [13]. Na OI há um déficit na codificação do colágeno do tipo I, o que impede o colágeno de exercer suas funções estruturais adequadamente. Além de sujeita-lo à degradação intra e extracelular, afetando a migração e diferenciação celular e, concomitantemente, a interação célula-matriz. Por conseguinte, estas alterações são consideradas o principal fator fisiopatológico da OI [21]. 


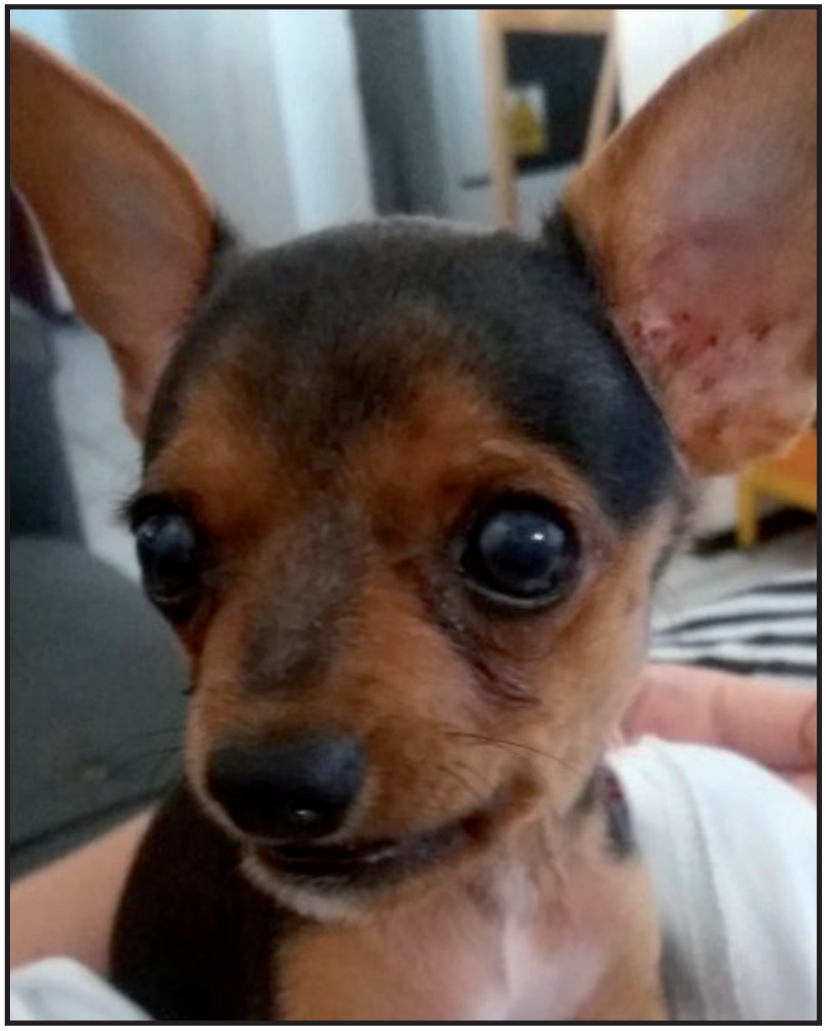

Figura 1. Cão da raça pinscher de 1 ano apresentando aspecto triangular da face e crânio abaulado bilateralmente.

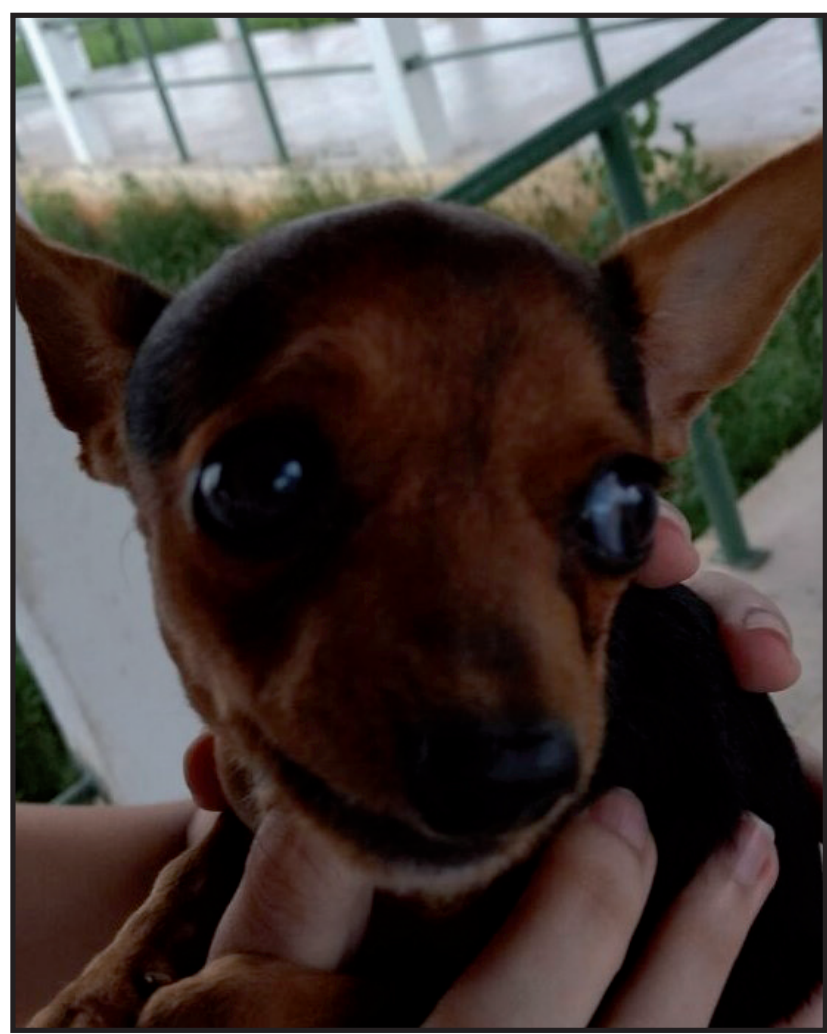

Figura 2. Cão da raça pinscher de 1 ano apresentando esclera azulada bilateralmente.

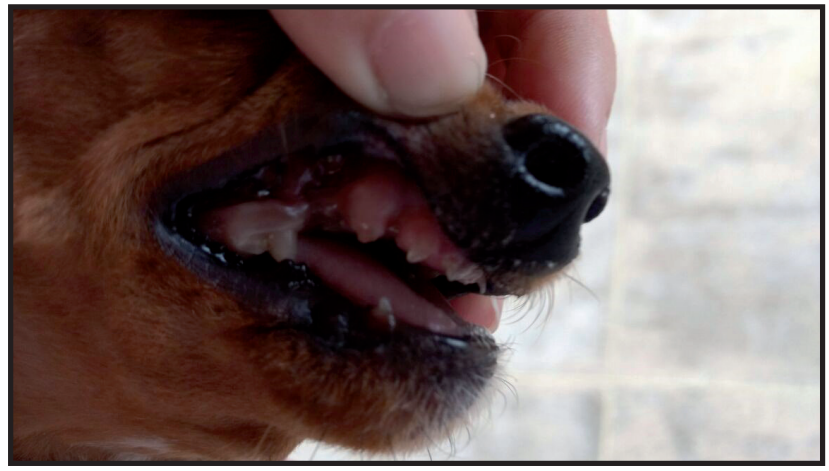

Figura 3. Cavidade bucal de cão da raça pinscher de 1 ano apresentando dentes pequenos, translúcidos, acinzentados, frágeis e deformados.

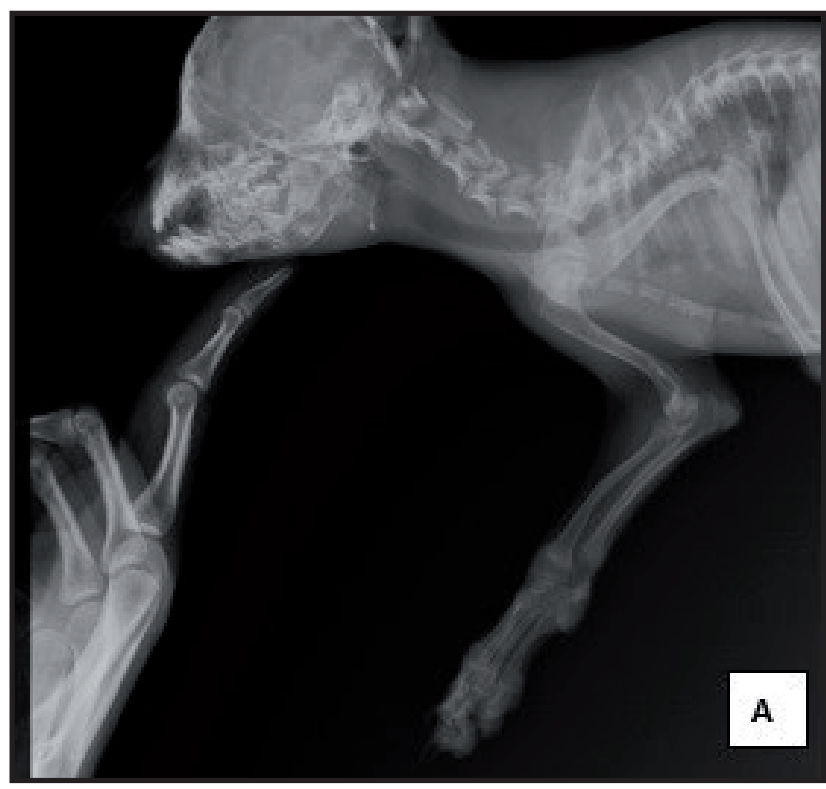

Figura 4. Exame radiográfico do crânio de cão da raça pinscher de 1 ano apresentando adelgaçamento da cortical com fontanelas persistentes e suturas cranianas abertas, denotando hidrocefalia.

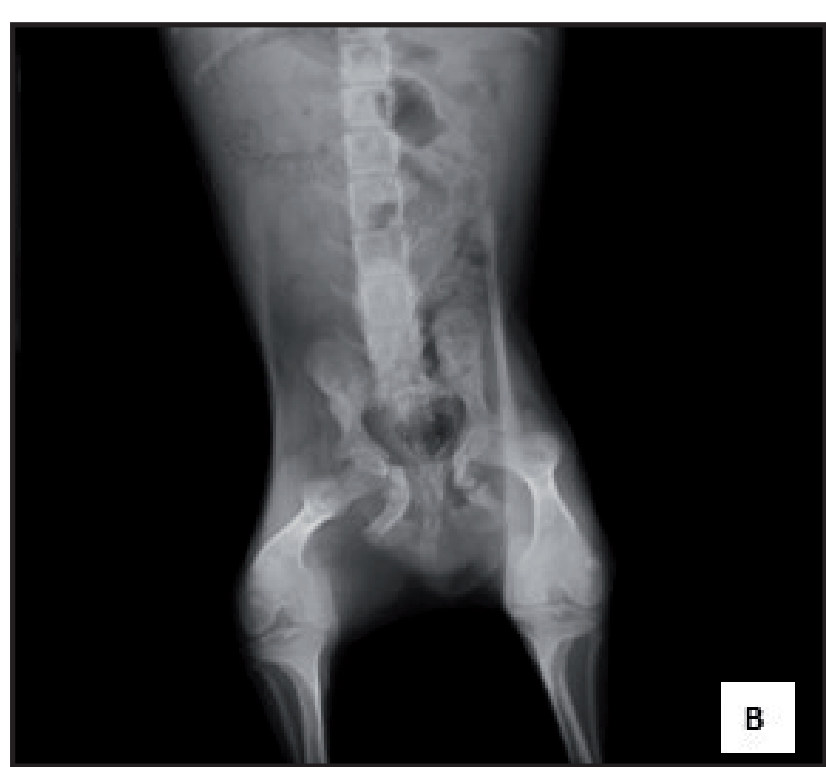

Figura 5. Exame radiográfico de abdomen de cão da raça pinscher de 1 ano, apresentando pelve e fêmur.com decréscimo generalizado na radiopacidade óssea, com adelgaçamento da cortical e múltiplas fraturas. 
No presente relato, não houveram alterações hematológicas, apenas um discreto aumento sérico na dosagem de cálcio $(12,3 \mathrm{mg} / \mathrm{dL})$. Estes achados colaboram principalmente com o que é descrito sobre a osteogênese imperfeita humane tipo VI [11]. Este fator imputa maior importância para métodos de diagnóstico histopatológico.

O diagnóstico foi efetuado primariamente levando-se em consideração dados chamativos como: fraturas de repetição, face triangular, crânio abaulado, escleras azuladas e opacidade de córnea [15]. Os exames radiográficos serviram para confirmação diagnóstica e controle das fraturas. Embora o teste genético forneça uma melhor compreensão da doença, deixou-se de realizá-lo no paciente, porquanto indisponível como rotina em muitos centros de tratamento [7].

A ampla variabilidade fenotípica é observada em indivíduos com OI; no entanto, há um padrão de fraturas e de características que ajudam a caracterizar clínica e radiologicamente a doença [3] e que constituem a base para a identificação de casos e o diagnóstico de OI.

A fragilidade óssea e suscetibilidade a fraturas com nenhum ou com mínimo trauma são características clássicas da OI [7], como o observado no histórico do paciente.

A fragilidade óssea é um sinal indicativo da OI [3]. No exame radiográfico do paciente foram visualizadas fraturas múltiplas na pelve, em nível de asa do íleo direito, corpo do ísquio esquerdo e corpo do ísquio direito; além da formação de calos ósseos opacos, metáfise da tíbia direita (alargada e angulada) e esclerose medular, corroborando achados descritos por Thrall [22]. Por conseguinte, fraturas de ossos longos, principalmente em membros inferiores, são as mais frequentes em pacientes com OI [19].

A face com o formato triangular, decorrente do calvário protuberante, o adelgaçamento da cortical com fontanelas persistentes e suturas cranianas abertas (hidrocefalia), assim como esclera azulada, devido a camada de colágeno encontrar-se mais fina, permitindo a visibilidade do pigmento intraocular conforme já descrito na literatura[20].

A DI, caracterizada por dentes translúcidos e escurecidos, de coloração que varia entre o cinzento, semelhante ao do paciente em questão, o amarelo ou o castanho, causa defeitos estruturais na formação na dentição decídua e permanente [1], porquanto a dentina é formada em grande parte por colágeno tipo I [6]. Contudo defeitos estruturais como raízes dentais estreitas e curtas com obliteração pulpar parcial também estão presentes na DI como observado anteriormente [23].

De acordo com Sillence et al. [20], a classificação do paciente se enquadra na OI tipo III, pois o mesmo apresenta deformação progressiva de membros, fraturas múltiplas (pelve e tíbia), fraqueza muscular, limitação marcante de mobilidade (perda dos movimentos das patas traseiras) e retardo severo de crescimento. Além de, hidrocefalia, escleras azuladas e DI.

Como diagnóstico diferencial devem ser consideradas várias condições que aumentam o risco de fraturas, bem como doenças que se assemelham à OI, sobrepondo características clínicas, tais como doença metabólica óssea da prematuridade, osteoporose juvenil idiopática, hiperparatireoidismo secundário a IRC [7]. No entanto, o animal em questão apesentou uma variedade de sinais clínicos, além das fraturas, que não pertencem a estas doenças, como esclera azulada e face triangular seguida de hidrocefalia e DI, confirmando o diagnóstico de OI.

Ainda não existe terapêutica medicamentosa curativa para a OI, porém várias outras medidas têm sido relacionadas com a melhora da sobrevida e da expectativa de vida dos pacientes. Pode-se destacar a importância dos bisfosfonatos, especialmente o pamidronato ou alendronato, com a diminuição da taxa de reabsorção, conduzindo ao aumento da densidade óssea, em humanos [21].

Do exposto se conclui que a osteogênese imperfeita é uma doença congênita de caráter debilitante, o que resulta em prognostico reservado.

Em animais com fragilidade óssea, deve ser realizado estudo clínico e radiográfico para o diagnóstico de OI. Ademais, por ser rara e de cunho genético, animais com OI ou suspeitos não devem ser utilizados como reprodutores.

\section{MANUFACTURER}

${ }^{1}$ Ouro Fino Saúde Animal Participações S.A. Cravinhos, SP, Brazil

Declaration of interest. The authors report no conflicts of interest. The authors alone are responsible for the content and writing of the paper. 


\section{REFERENCES}

1 Abukabbos H. \& Al-Sineedi F. 2013. Clinical manifestations and dental management of dentinogenesis imperfecta associated with osteogenesis imperfecta: Case report. Saudi Dentistry Journal. (25): 159-165.

2 Agerholm J.S., Lund A.M., Bloch B., Reibel J., Basse A. \& Arnbjerg J. 1994. Osteogenesis imperfecta in HolsteinFriesian calves. Transboundary and Emerging Diseases. 41(1-10): 128.

3 Albright J.A. \& Grunt J.A. 1971. Studies of patients with osteogenesis imperfecta. Journale Bone Joint SurgeryAmerican. 53(1): 1415-1425.

4 Arthur D.G., Thompson K.G \& Swarbrick P. 1992. Lethal osteogenesis imperfecta and skin fragility in newborn New Zealand Romney lambs. New Zealand Veterinary Journal. 40(3): 112-116.

5 Åstrom E. \& Soderhall S. 1998. Beneficial effect of bisphosphonate during five years of treatment of severe osteogenesis imperfecta. Acta Paediatrica. 87(1): 64-68.

6 Bellary S.S., Steinberg A., Mirzayan N., Shirak M., Tubbs R.S. \& Cohena-Gadol A.A. \& Loukas M. 2013. Wormian bones: a review. Clinical Anatomy. 26(1): 922-927.

7 Brizola E., Zambrano M.B., Pinheiro B.S., Vanz A.P. \& Félix T.M. 2017. Clinical features and pattern of fractures at the time of diagnosis of osteogenesis imperfecta in children. Revista Paulista de Pediatria. 35(1): 171-177.

8 Campbell B.G., Wootton J.A., MacLeod J.N. \& Minor R.R. 2011. Canine COL1A2 mutation resulting in C-terminal truncation of pro-alpha2 (I) and severe osteogenesis imperfecta. Journal of Bone and Mineral Research. 16(6): 1147-1153.

9 Campbell B.G., Wootton J.A., MacLeod J.N. \& Minor R.R. 2000. Sequence of normal canine COL1A1 cDNA and identification of a heterozygous alpha1(I) collagen Gly208Ala mutation in a severe case of canine osteogenesis imperfecta. Archives of biochemistry and biophysics. 384(1): 37-46.

10 Chipman S.D., Sweet H.O. \& McBride D.J. 1993. Defective pro alpha 2 (I) collagen synthesis in a recessive mutation of mice: a model of human osteogenesis imperfecta. Proceedings of the National Academy of Sciences. 90(5): 1701-1705.

11 Glorieux F.H., Ward L.M., Rauch F., Lalic L., Roughley P.J. \& Travers R. 2002. Osteogenesis imperfecta type VI: a form of brittle bone disease with a mineralization defect. Journal of Bone and Mineral Research. 17: 30-38.

12 Horvarth S.A., Francestri F.L. \& Riveross V. 1986. Treatment of osteogenesis imperfecta in a tiger (Panthera tigris). Av Cienc Vet. 1(1): 49-51.

13 Huber M.A. 2007. Osteogenesis imperfecta. Oral Surgery, Oral Medicine, Oral Pathology, Oral Radiology and Endodontics. 103(3): 314-320 .

14 Jones K. L., Jones M.C. \& Campo M.D.C. 2013. Smith's Recognizable Patterns of Human Malformation. 7th edn. Philadelphia: Saunders, pp.634-638 .

15 Kanno C.M. \& Oliveira J.A. 2009. Clinical and radiographic features of osteogenesis imperfecta associated with dentinogenesis imperfecta. Revista Odonto. 17(33): 96-101.

16 Rauch F. \& Glorieux F.H. 2004. Osteogenesis imperfecta. Lancet. 363(9418): 1377-1385.

17 Schmidt V. 1967. Osteogenesis imperfecta in 2 collie litter siblings. Wiener tierarztliche Monatsschrift. 54(2): 92.

18 Seeliger F., Leeb T., Peters M., Brugmann M., Fehr M. \& Hewicker-Trautwein M. 2003. Osteogenesis imperfecta in two litters of dachshunds. Veterinary Pathology. 40(5): 530-539.

19 Shapiro J.R. \& Sponseller P.D. 2009. Osteogenesis imperfecta: questions and answers. Current opinion in pediatrics. 21(6): 709-716.

20 Sillence D.O., Senn A. \& Danks D.M. 1979. Genetic heterogeneity in osteogenesis imperfecta. Journal of Medical Genetics. 16(1): 101-116.

21 Souza A.S.R., Cardoso A.S., Lima M.M.S. \& Guerra G.V.Q.L. 2006. Prenatal diagnosis and vaginal delivery in osteogenesis imperfecta: a case report. Revista Brasileira de Ginecologia e Obstetrícia. 28: 244-250.

22 Thrall D.E. 2015. Textbook of Veterinary Diagnostic Radiology. 6th edn. Philadelphia: Elsevier Inc., 275p.

23 Wieczorek A., Loster J., Ryniewicz W., Ryniewicz A.M. \& Ryniewicz A.M. 2013. Dentinogenesis imperfecta hardness and Young's modulus of teeth. Acta of Bioengineering and Biomechanics. 15: 65-69.

24 Won S., Chung W.J. \& Yoon J. 2017. Clinical application of quantitative computed tomography in osteogenesis imperfecta-suspected cat. Journal of Veterinary Science. 18(3): 415-417. 